\title{
TG03
}

\section{Integrated Multi-domain Field Development Process to Unlock Middle East Tight Gas Reservoirs}

\author{
A. Aly* (Schlumberger) \& L. Ramsey (Schlumberger)
}

We will present the challenges of the developments in this emerging environment where the services and the technology have not matured yet to the North America levels.

Key conclusion of the paper is that by applying the multi-domain integrated field management process in both environments (North America \& Middle East) has resulted in significant improvements in production optimization, as well as single-well and field development planning. 
As gas demand rises and operators turn to tight gas reservoirs for new supplies, the need to optimize the capacity and recovery potential from this type of reservoir has risen. The BP statistical review of world energy 2008 highlights the volumes of proved natural gas reserves at the end of 2007 and it shows that most of the gas volumes (including tight gas) reside in the Middle East. Now we need to shift our focus on how to develop those gas reservoirs in the Middle East and adapt the processes and techniques developed in other regions of the world especially in North America to the Middle East challenging reservoirs.

In this paper we will present a multi-domain integrated process that enables the data and activities from multiple domains to be integrated from the single-well completion optimization level all the way to the field (geocellular and simulation modeling) level. Through this process various development scenarios for completions and drilling locations can be systematically and rigorously analyzed and optimized.

We will present two case studies from North America and the Middle East to show the applications of this process in 2 different environments (Mature and Emerging). The case from North America illustrates using the proposed process in a high volume field development and the value of applying new technology to optimize the process and reach a "SMART" factory like process that will allow integration of new technology and adaptive modification to the factory mode to get the optimal development process that will result in the highest production recoveries while maximizing the net present value from the asset.

While the Case from the Middle East shows the application of this process in the Mushayb field tight gas Qasim reservoir in Saudi Arabia. The challenge in this case is in collecting the needed \& the adequate data early in the life of the field to plan for an optimal development. We can show the value of collecting the key data early in a complex tight gas environment and how it can impact the development later and result in costly decisions. We will show the multi-domain work performed to optimize the well configuration for Qasim reservoir in order to maximize the final recovery factor. We investigated the optimal well type (vertical well with Frac and horizontal well), number of stage Frac and Frac half length, sensitivities on BHP for different scenarios and comparison between compositional (condensate Gas) and the dry gas simulations.

We will present the challenges of the developments in this emerging environment where the services and the technology have not matured yet to the North America levels.

Key conclusion of the paper is that by applying the multi-domain integrated field management process in both environments (North America \& Middle East) has resulted in significant improvements in production optimization, as well as single-well and field development planning. 